\title{
Implantes posextracción con regeneración ósea guiada
}

\author{
Postextraction implants with guided bone regeneration
}

\author{
Guiselle Andrea Verástegui Baldárrago ${ }^{1}$ \\ ${ }^{1}$ Facultad de Odontología de la Universidad Latinoamericana CIMA. Tacna, Perú. \\ ${ }^{a}$ Docente, Magister en Odontoestomatología.
}

\section{Resumen}

La colocación de implantes en alveolos post-extracción regenerados es un tipo de tratamiento que ha dado buenos resultados postratamiento. Los factores clave para conseguir el éxito en este tipo de tratamientos se basa en realizar una extracción atraumática del diente para preservar el hueso alveolar, ausencia de infección activa y estabilidad primaria del implante. Este caso clínico describe a una paciente de 40 años de edad que se realizó la extracción de la pieza 2.5 y la colocación de un implante inmediato con regeneración ósea guiada. Una vez aplicada la técnica y el período posoperatorio, fue evidente la buena oseointegración del implante a los 3 meses y una mejoría en la estética y soporte perimplantario. Sin duda, la tasa de éxito para los implantes colocados posexodoncia era comparable con aquella en áreas edéntulas.

Palabras clave: Implante inmediato, posexodoncia, regeneración ósea guiada.

\begin{abstract}
The placement of implants in regenerated postextraction alveoli is a type of treatment that has given good posttreatment results. The key factors to achieve success in this type of treatment are based on performing an atraumatic extraction of the tooth to preserve the alveolar bone, absence of active infection and primary implant stability. This clinical case describes a 40 -year-old patient who had the extraction of part 2.5 and the placement of an immediate implant with guided bone regeneration. Once the technique and the postoperative period were applied, the effective osseointegration of the implant was evident after 3 months, as well as an improvement in aesthetics and perimplant support. Undoubtedly, the success rate for implants placed postexodontics was comparable with that in edentulous areas.
\end{abstract}

Keywords: Immediate implant, postextraction, guided bone regeneration. 


\section{Introducción}

Los implantes dentales se han convertido en una técnica de rutina donde los resultados son muy predecibles y están basados en protocolos quirúrgicos y prostodónticos con altas tasas de éxito. A finales de los años 70, en la Universidad de Tübingen, Schulte (1) se propugnaba la técnica de implantes inmediatos. Este tipo de técnica no solo está indicada para la substitución de dientes con patologías sin posibilidad de tratamiento, también se utiliza en la simultánea extracción de caninos incluidos, dientes temporales y, en los casos, donde haya lesiones apicales crónicas que no mejoran después de la endodoncia y cirugía periapical $(2,3)$.

La colocación de implantes post extracción ofrece una serie de ventajas, como la reducción de las intervenciones quirúrgicas (4). Con respecto a las regiones posteriores, la colocación inmediata del implante implica una serie de problemas clínicos referente a los aspectos anatómicos del sitio. El espacio implante-alvéolo puede llegar a ser una desventaja si no se obtiene una estabilidad primaria inicial, por lo tanto, la colocación inmediata presenta desafíos para la osteointegración. Sin embargo, varias técnicas y materiales han sido desarrollados y utilizados para aumentar la formación de hueso y la osteointegración, tales como la regeneración ósea guiada $(5,6)$. Novaes et al. (7) realizaron un estudio en perros colocando implantes inmediatos en localizaciones con infección crónica periapical. Al finalizar, refirieron buenos resultados y demostraron que, a pesar de los signos evidentes de patología periapical, no está contraindicada la colocación de implantes si se administra un tratamiento antibiótico pre y posoperatorio y se realizaba una adecuada limpieza del lecho alveolar previo a la implantación.

El objetivo de este reporte de caso es mostrar el manejo clínico y la evolución clínica de un caso de implante de instalación inmediata; basándose en la literatura y enfocándose en el mejor manejo posible para el paciente y obtener un resultado predecible para lograr el éxito y/o supervivencia del implante en boca.

\section{Caso clínico}

Paciente de 50 años de edad, de sexo femenino, ASA I acude a consulta en mayo del 2018. Al realizar la historia clínica el paciente presenta buen estado general. Al examen clínico, la pieza dentaria presenta fractura de la corona con movilidad dentaria grado II. Al examen radiográfico presenta un absceso dentoalveolar crónico con reabsorción ósea a nivel apical (Figura 1).

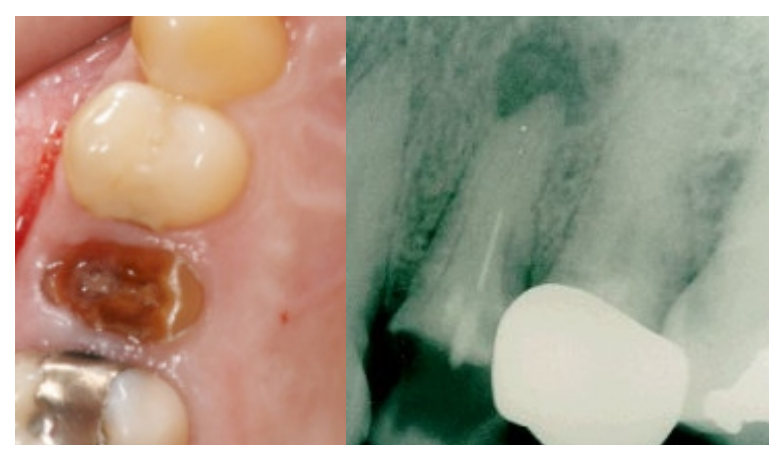

Figura 1. Preoperatoria/Radiografía Periapical.

El paciente fue notificado de las opciones de tratamiento, a primera instancia, el paciente optó por extraerse el diente y dejar que cicatrice. Al día siguiente de la extracción, se cambió de opinión y se decidió por el tratamiento con implante; luego de firmar el consentimiento informado, se procedió a realizar los siguientes tratamientos: Fase I: Educación y motivación, instrucciones de higiene oral, destartraje. Fase 2: Extracción de la pieza 2.5 (previamente realizada un día anterior), colocación de implante dental en espacio edéntulo. Fase 3: Regeneración ósea guiada alrededor del implante. Fase 4: Colocación del cicatrizal. Luego de 5 meses posterior a la colocación del implante dental y valorando el estado de oseointegración, no se reportaron complicaciones y la cicatrización ósea fue apropiada. Fase 5: Colocación de corona atornillada definitiva.

\section{Procedimiento quirúrgico}

Los días previos a la cirugía, el paciente comienza un régimen de antibiótico preventivo (amoxicilina $500 \mathrm{mg}$ ) durante una semana. Se realizó la asepsia y antisepsia del campo operatorio extraoral con yodopovidona, e intraoral con gluconato de clorhexidina al $0.12 \%$. Se aplicó anestesia local infiltrativa con lidocaína al $2 \%$ y epinefrina 1:5000. La pieza 2.5 fue removida quirúrgicamente de manera atraumática respetando las tablas óseas (Figura 2).

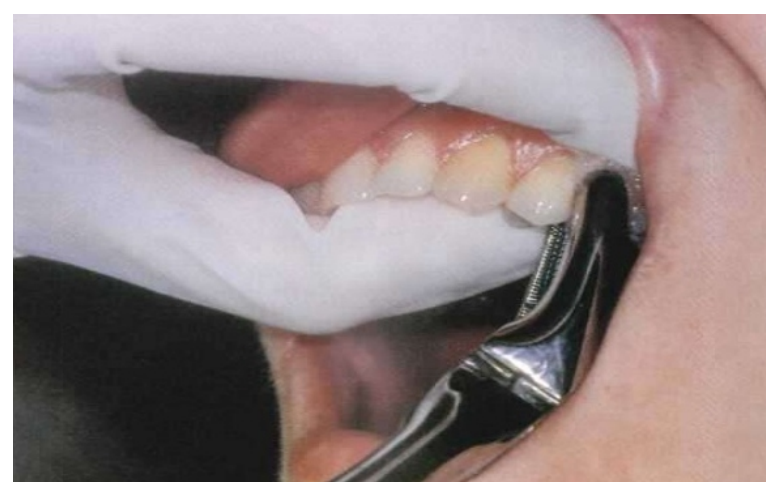

Figura 2. Extracción de la pieza 
Al examen tomográfico con guía quirúrgica, se estableció diámetro y altura adecuada para instalar implante dental (Figura 3a). Con una sonda periodontal se verificó la integridad de las paredes del alveolo y determinar las dimensiones del implante y la cantidad de hueso a utilizar. Incisión: En la zona posterior, se realizó un levantamiento de colgajo a espesor total con incisiones con descargas verticales.

Colocación del implante de conexión interna de forma sumergida (Neobiotech $4 \times 10.5 \mathrm{~mm}$ ) colocado a $1 \mathrm{~mm}$ por detrás de la tabla vestibular de la cresta alveolar y se utilizó un torque quirúrgico de $30 \mathrm{Ncm}$, para mejorar la estabilidad primaria del implante (Figura 3b).

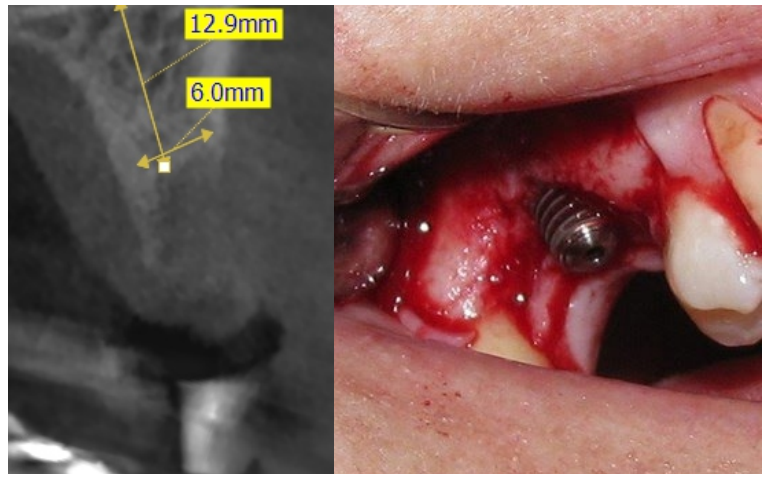

Figura 3a, 03b. Corte Tomográfico/Colocación del Implante.

Una vez instalado el implante, debido al defecto óseo a nivel vestibular, se recorta y adapta la membrana reabsorbible estabilizándola por vestibular. Se hidrata el relleno óseo fosfato tricálcico en suero fisiológico y se condensa en el defecto óseo, para luego reposicionar la membrana reabsorbible de colágeno hacia vestibular (Figura 4) y el colgajo.

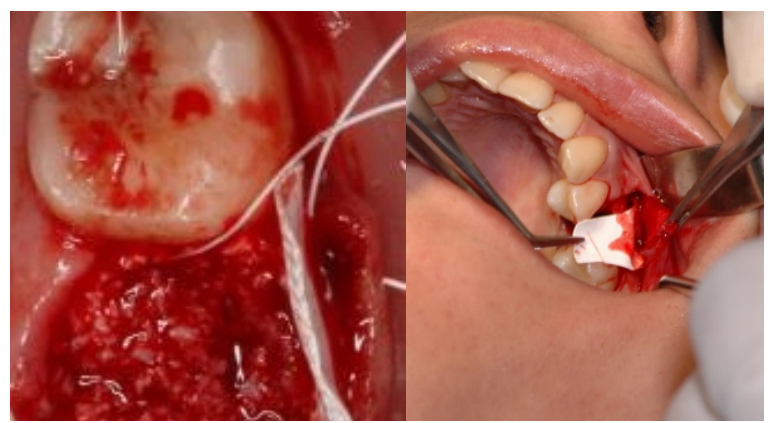

Figura 4. Regeneración ósea guiada.

Se sutura el colgajo sin tensión con ácido poliglicólico 4 / 0, puntos suspensorios laterales (Figura $5)$.

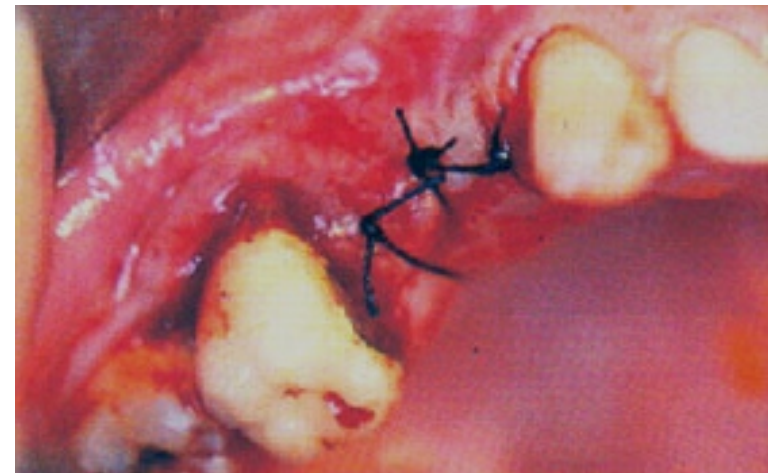

Figura 5. Sutura.

\section{Seguimiento clínico}

Al examen radiográfico a los 10 días posoperatorio, no se encontró complicaciones; se observó un tejido en proceso de cicatrización. A los 5 meses, luego de la colocación del implante, se le colocó el pilar de cicatrización. Luego, se realizó la carga funcional de los implantes mediante la colocación de la correspondiente prótesis implantosoportada, corona atornillada sobre pilar UCLA semicalcinable con base metálica (Figura 6).

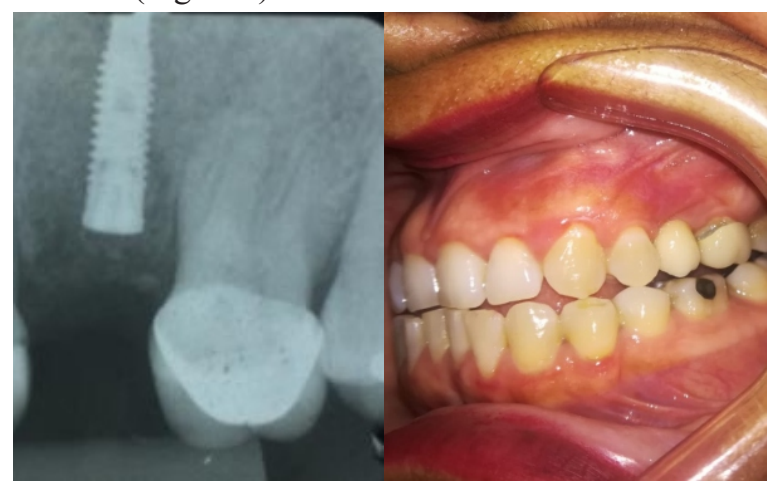

Figura 6. Posoperatorio.

\section{Discusión}

En los últimos años, se ha introducido en la práctica clínica una técnica que consiste en la colocación de implantes de forma inmediata a la extracción, denominada implante posextracción. Este procedimiento tiene buenos resultados clínicos, según muchas investigaciones, con un éxito que va desde el 92.7 al $98 \%(8,9)$. Grunderr et al. (10), por ejemplo, no encontraron diferencias significativas respecto al éxito de supervivencia a largo plazo entre implantes inmediatos $(92.4 \%)$ y diferidos $(94.7 \%)$.

La colocación de un implante posextracción, de acuerdo a los reportes obtenidos nos muestra que es una técnica que nos ayuda a preservar el volumen de los tejidos blandos y duros, evitando así la perdida de 
anchura y altura crestal (11). Sin embargo, en casos de atrofia alveolar puede limitar los resultados estéticos y funcionales de la rehabilitación protésica implanto soportadas (12). La instalación del implante debe ser guiada por la restauración protética y no por el hueso disponible (13). La arquitectura ósea determina el contorno y la posición del tejido blando periimplantar. En consecuencia, el volumen tridimensional del reborde alveolar es un factor determinante para un buen resultado estético (14). Las técnicas de regeneración ósea guiada (ROG) representan una alternativa para la obtención de resultados estéticos satisfactorios en la regeneración de defectos peri-implantares (15).

En conclusión, el tratamiento con implantes en alveolos posextracción ha reducido los tiempos de tratamiento, minimizando la pérdida ósea y conservando la estructura gingival. Este es un procedimiento con alta tasa de supervivencia cuya principal ventaja es acortar el tiempo de tratamiento. Al igual que otras técnicas se necesita un estudio adecuado de cada caso y el seguimiento de estos es aún breve, razón por la cual se requieren estudios más exhaustivos.

\section{Referencias bibliográficas}

1. Schulte W. The intra-osseous A1203 (Frialit) Tübingen implant. Developmental status after eight years (II). Quintessence Int 1984; 154: 19-35.

2. Calvo JL, Muñoz EJ. Implantes inmediatos oseointegrados como reemplazo a caninos superiores retenidos. Evaluación a 3 años. Rev Europea Odontoestomatol 1999; 6:313-20.

3. Coppel A, Prados JC, Coppel J. Implantes postextracción: situación actual. Gaceta Dental Sept. 2001; 120: 80-6.

4. Lazzara RJ. Immediate implant placement into extraction sites: surgical and restorative advantages. Int J Periodontics Restorative Dent. 1989; 9: 332343.

5. Atieh MA, Payne AG, Duncan WJ, de Silva RK, Cullinan MP. Immediate placement or immediate restoration/loading of single implants for molar tooth replacement: A systematic review and metaanalysis. Int J Oral Maxillofac Implants. 2010; 25: 401-415.

6. Paolantonio M, Dolci M, Scarano A, d'Archivio D, di Placido G, Tumini V et al. Immediate implantation in fresh extraction sockets. A controlled clinical and histological study in man. J Periodonto. 2001; 72: 1560-1571.

7. Novaes AB, Vidigal GM, Novaes AB, Grisi MF, Polloni S, Rosa A. Immediate implants placed into infected sites. A histomorphometric study in dogs. Int J Oral Maxillofac Implants 1998; 13: 422-7.

8. Gelb DA. Immediate implant surgery: three-year retrospective evaluation of 50 consecutives cases. Int J Oral Maxillofac Implants 1993; 8: 388-99.
9. Krump JL, Barnet BG. The immediate implant: a treatment alternative. Int J Oral Maxillofac Implants 1991; 6: 19-23.

10. Grunder U, Polizzi G, Goene R, Hatano N, Henry P, Jackson WJ et al. A 3 year prospective multicenter follow-up report on the immediate and delayed immediate placement of implants. Int $\mathrm{J}$ Oral Maxillofac Implants 1999; 14: 210-6.

11. Carlsson GE, Persson G. Morphologic changes of the mandible after extraction and wearing of dentures. A longitudinal, clinical, and x-ray cephalometric study covering 5 years. Odontol Revy 1967; 18: 27-54.

12. Schropp L., Wenzel A., Kostopoulos L., Karring T.: Bone healing and soft tissue contour changes following single-tooth extraction: a clinical and radiographic 12-month prospective study. Int $\mathrm{J}$ Periodontics Restorative Dent (2003); 23(4): 31323.

13. Chan H.L., Garaicoa-Pazmino C., Suarez F., Monje A., Benavides E., Oh T.J., Wang H.L.: Incidence of implant buccal plate fenestration in the esthetic zone: a cone beam computed tomography study. Int J Oral Maxillofac Implants (2014); 29(1): 171-7.

14. Benic G.I., Ge Y., Gallucci, G.O., Jung, R.E., Schneider, D., Hammerle, C.H.: Guided bone regeneration and abutment connection augment the buccal soft tissue contour: 3-year results of a prospective comparative clinical study. Clin Oral Implants Res (2017); 28(2): 219-225.

15 Seibert J.S.: Treatment of moderate localized alveolar ridge defects. Preventive and reconstructive concepts in therapy. Dent Clin North $\operatorname{Am}(1993) ; 37(2): 265-80$.
Correspondencia:

andrea_gis_4@hotmail.com
Fecha de recepción : 03 de agosto de 2019

Fecha de aceptación : 15 de noviembre de 2019 